\title{
Review Article \\ Emerging Molecularly Targeted Therapies in Castration Refractory Prostate Cancer
}

\author{
Jesal C. Patel, ${ }^{1}$ Benjamin L. Maughan, ${ }^{2}$ Archana M. Agarwal, ${ }^{3}$ Julia A. Batten, \\ Tian Y. Zhang, ${ }^{2}$ and Neeraj Agarwal ${ }^{1}$ \\ ${ }^{1}$ Division of Medical Oncology, University of UT Huntsman Cancer Institute, Salt Lake City, Utah 84112, USA \\ ${ }^{2}$ Department of Internal Medicine, University of UT, Salt Lake City, Utah 84112, USA \\ ${ }^{3}$ Department of Pathology and ARUP Laboratories, University of UT, Salt Lake City, Utah 84108, USA
}

Correspondence should be addressed to Neeraj Agarwal; neeraj.agarwal@hci.utah.edu

Received 31 January 2013; Accepted 10 April 2013

Academic Editor: William L. Dahut

Copyright (C) 2013 Jesal C. Patel et al. This is an open access article distributed under the Creative Commons Attribution License, which permits unrestricted use, distribution, and reproduction in any medium, provided the original work is properly cited.

\begin{abstract}
Androgen deprivation therapy (ADT) with medical or surgical castration is the mainstay of therapy in men with metastatic prostate cancer. However, despite initial responses, almost all men eventually develop castration refractory metastatic prostate cancer (CRPC) and die of their disease. Over the last decade, it has been recognized that despite the failure of ADT, most prostate cancers maintain some dependence on androgen and/or androgen receptor (AR) signaling for proliferation. Furthermore, androgen independent molecular pathways have been identified as drivers of continued progression of CRPC. Subsequently, drugs have been developed targeting these pathways, many of which have received regulatory approval. Agents such as abiraterone, enzalutamide, orteronel (TAK-700), and ARN-509 target androgen signaling. Sipuleucel-T, ipilimumab, and tasquinimod augment immunemediated tumor killing. Agents targeting classic tumorogenesis pathways including vascular endothelial growth factor, hepatocyte growth factor, insulin like growth factor-1, tumor suppressor, and those which regulate apoptosis and cell cycles are currently being developed. This paper aims to focus on emerging molecular pathways underlying progression of CRPC, and the drugs targeting these pathways, which have recently been approved or have reached advanced stages of development in either phase II or phase III clinical trials.
\end{abstract}

\section{Introduction}

Prostate cancer is the most common noncutaneous malignancy and one of the common causes of cancer related deaths among men in the United States [1]. The majority of men are diagnosed with early stage disease. However, approximately a third will eventually develop metastatic disease. The cornerstone of treatment for advanced disease is medical castration by androgen deprivation therapy with a gonadotropin releasing hormone agonist $(\mathrm{GnRH})$ or less commonly, surgical castration with orchiectomy. Despite initial responses, almost all patients will develop disease progression, a stage known as metastatic castration refractory prostate cancer (mCRPC). One of the reasons why prostate cancer continues to progress is the persistence of androgen receptor signaling, despite castrate level of androgens [2]. Additionally, there are androgen independent pathways responsible for prostate cancer progression. This paper summarizes the recent advancements in the development of therapies that target these molecular pathways in CRPC, with an emphasis on agents that are being evaluated in phase II or III clinical trials and those recently approved for the treatment of CRPC (Figure 1, Tables 1 and 2).

\section{Targeting Androgen Signaling Pathway}

Several mechanisms have been identified to explain persistent androgen signaling in CRPC $[2,3]$, including increased AR gene expression, mutation of the AR gene, or upregulation of enzymes involved in androgen synthesis [3-6]. Cytochrome P450 17 alpha-hydroxylase and C17,20-lyase (CYP17) are the critical enzymes for synthesis of androgens in the adrenal glands and prostate tumors [6]. 


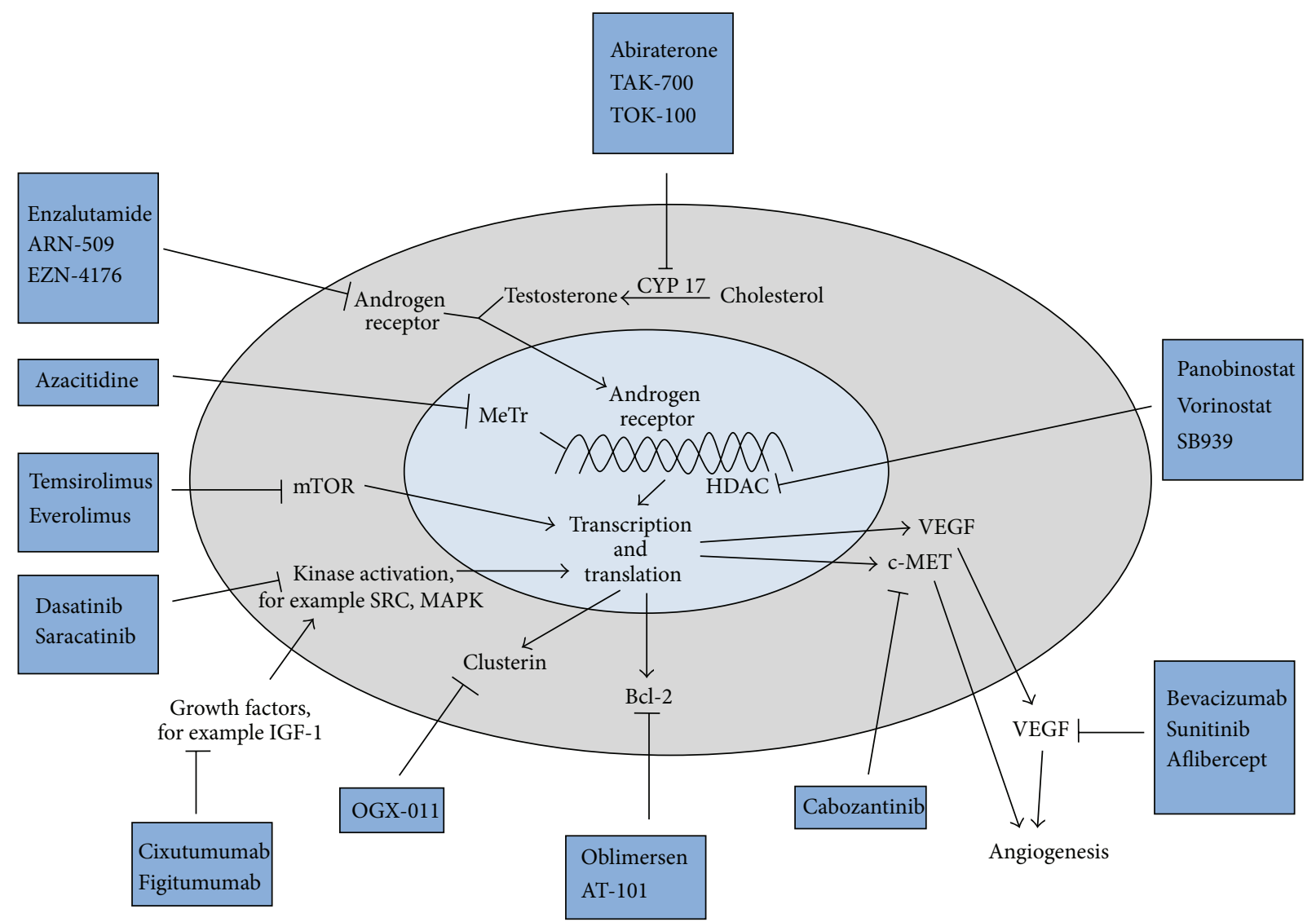

FIGURE 1: Androgen dependent and independent molecular pathways underlying progression of castration refractory prostate cancer and drugs targeting those pathways. CYP17: Cytochrome P450 17 alpha-hydroxylase and C17,20-lyase; mCRPC: metastatic castration refractory prostate cancer; AR: androgen receptor; OS: overall survival; PFS: progression free survival; c-MET: hepatocyte growth factor receptor; VEGF: vascular endothelial growth factor; MeTr: DNA methyl transferase; HDAC: histone deacetylases; Src: Src kinase; MAPK: MAP kinase; IGF-1: insulin like growth factor-1.

Abiraterone acetate, a pregnenolone analog, is an orally administered small molecule that irreversibly inhibits CYP17. Abiraterone was shown to be clinically beneficial and improved overall survival compared to placebo in a landmark phase III trial in patients who had been previously treated with docetaxel (14.8 months versus 10.9 months, $P<0.0001)$ [7]. Abiraterone was well tolerated with mild adverse effects associated with secondary mineralocorticoid excess. In 2011, the FDA approved the use of abiraterone for treatment of CRPC in patients who were previously treated with docetaxel. More recently, another phase III study showed improved radiographic PFS with abiraterone over placebo in men with CRPC who had never received chemotherapy, leading to the regulatory approval of abiraterone in prechemotherapy setting as well [8].

Orteronel (TAK-700), like abiraterone, is a novel inhibitor of the CYP17 pathway. However, TAK-700 more specifically inhibits CYP17, 20 lyase versus CYP17 hydroxylase, and does not generally lead to the syndrome of secondary mineralocorticoid excess. The updated data obtained from phase II portion of a phase I/II study of TAK-700 in chemonaive patients with metastatic CRPC were reported in 2012 Genitourinary Cancers Symposium [9]. Ninety-six chemonaive patients with metastatic CRPC were treated in four TAK-700 dose cohorts. The PSA reduction rates were favorable across all cohorts. Of the 51 patients who had RECIST-evaluable disease, 10 had a partial response, 22 had stable disease, and 15 had disease progression. In a phase II study of patients with nonmetastatic CRPC with biochemical recurrence, 38 patients were treated with TAK-700 at a dose of $300 \mathrm{mg}$ twice daily, without prednisone [10]. Treatment at this dose was feasible with manageable toxicities. After three months of treatment, $16 \%$ achieved PSA $\leq 0.2 \mathrm{ng} / \mathrm{Ml}, 76 \%$ achieved $\geq 50 \%$ decrease, and $32 \%$ achieved a PSA reduction of $\geq 90 \%$. Median time to PSA progression was 14.8 months. Currently, there are separate phase 3 trials evaluating TAK-700 in men with progressive CRPC, who are either chemotherapy naïve or posttreatment with docetaxel (Table 1).

Enzalutamide (MDV 3100) is a novel AR antagonist that binds to AR with an eight-times higher affinity than bicalutamide and reduces the efficiency of nuclear translocation of the androgen receptor, DNA binding to androgen response elements, and recruitment of coactivators by the androgen receptor [11]. In contrast to bicalutamide, MDV3100 has no known agonist activity when $\mathrm{AR}$ is overexpressed. In a phase 


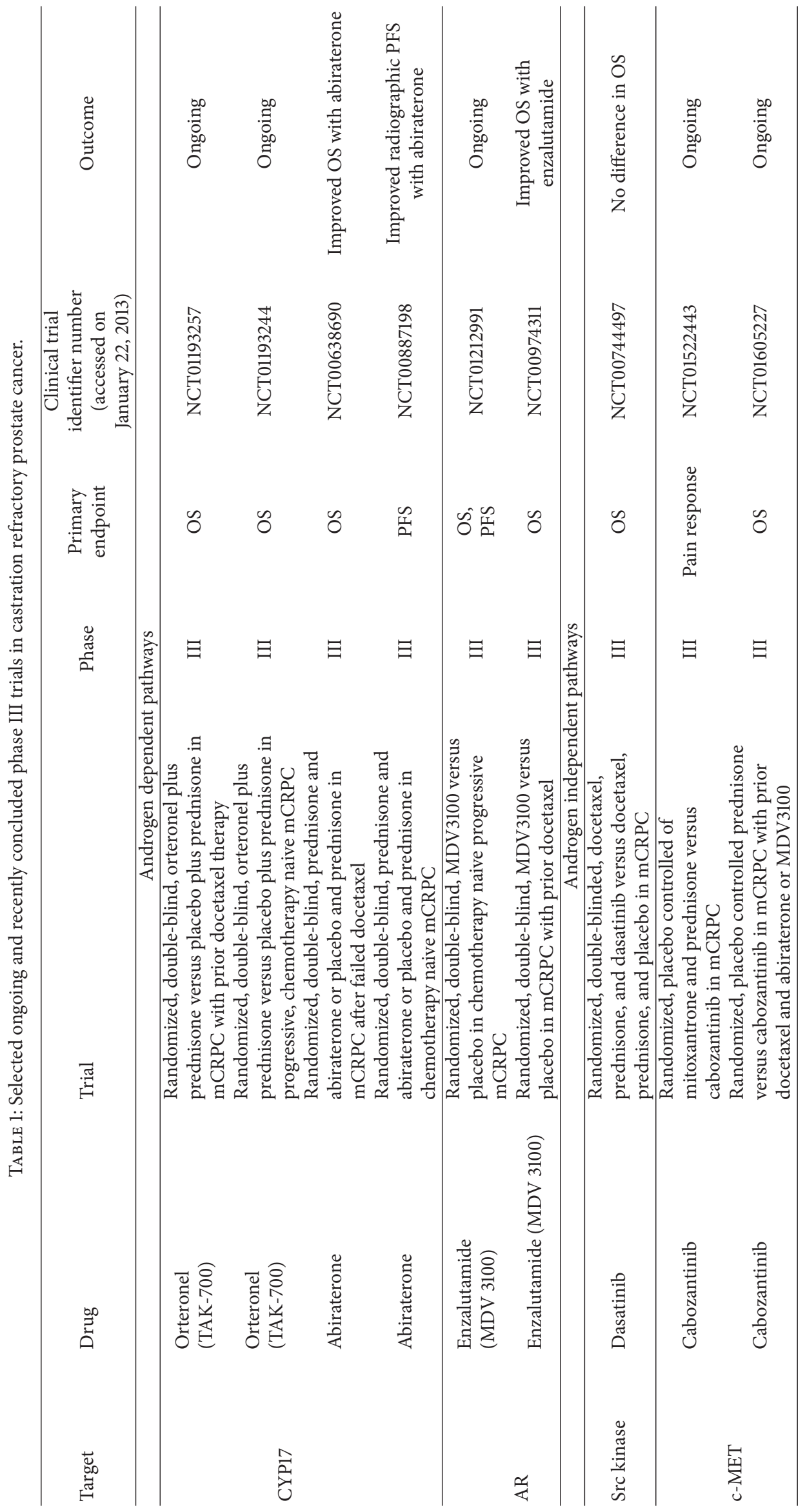




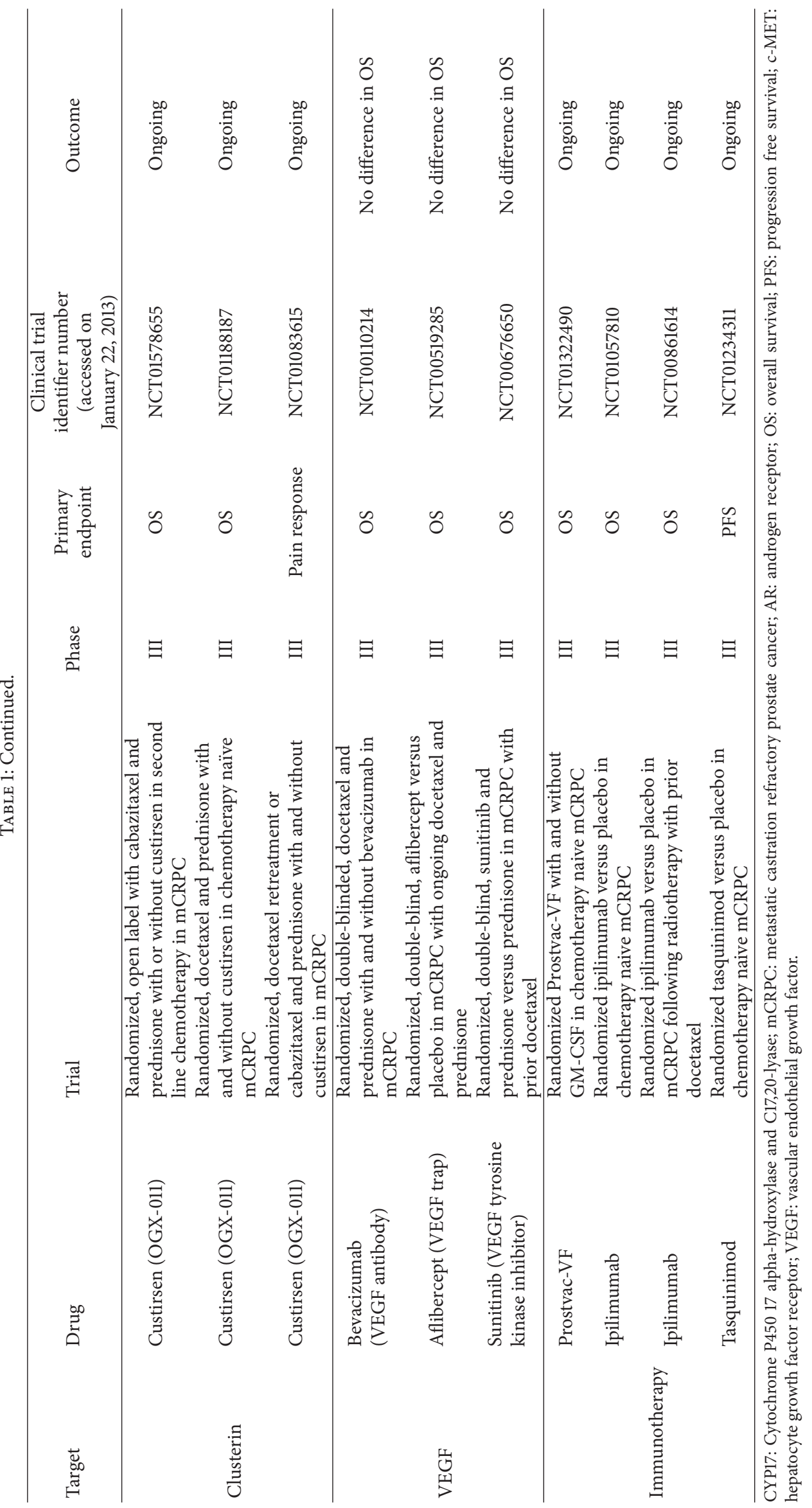




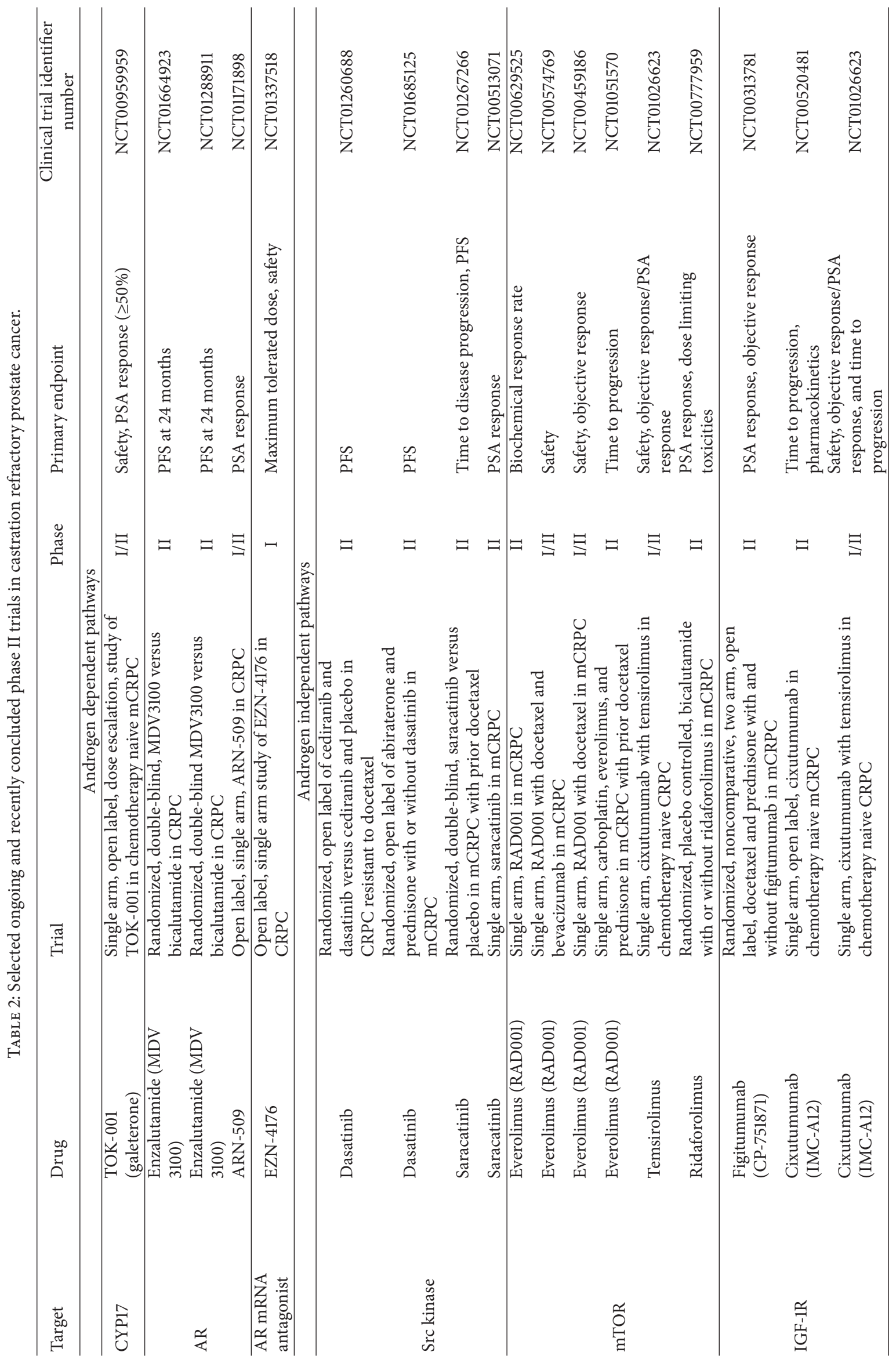




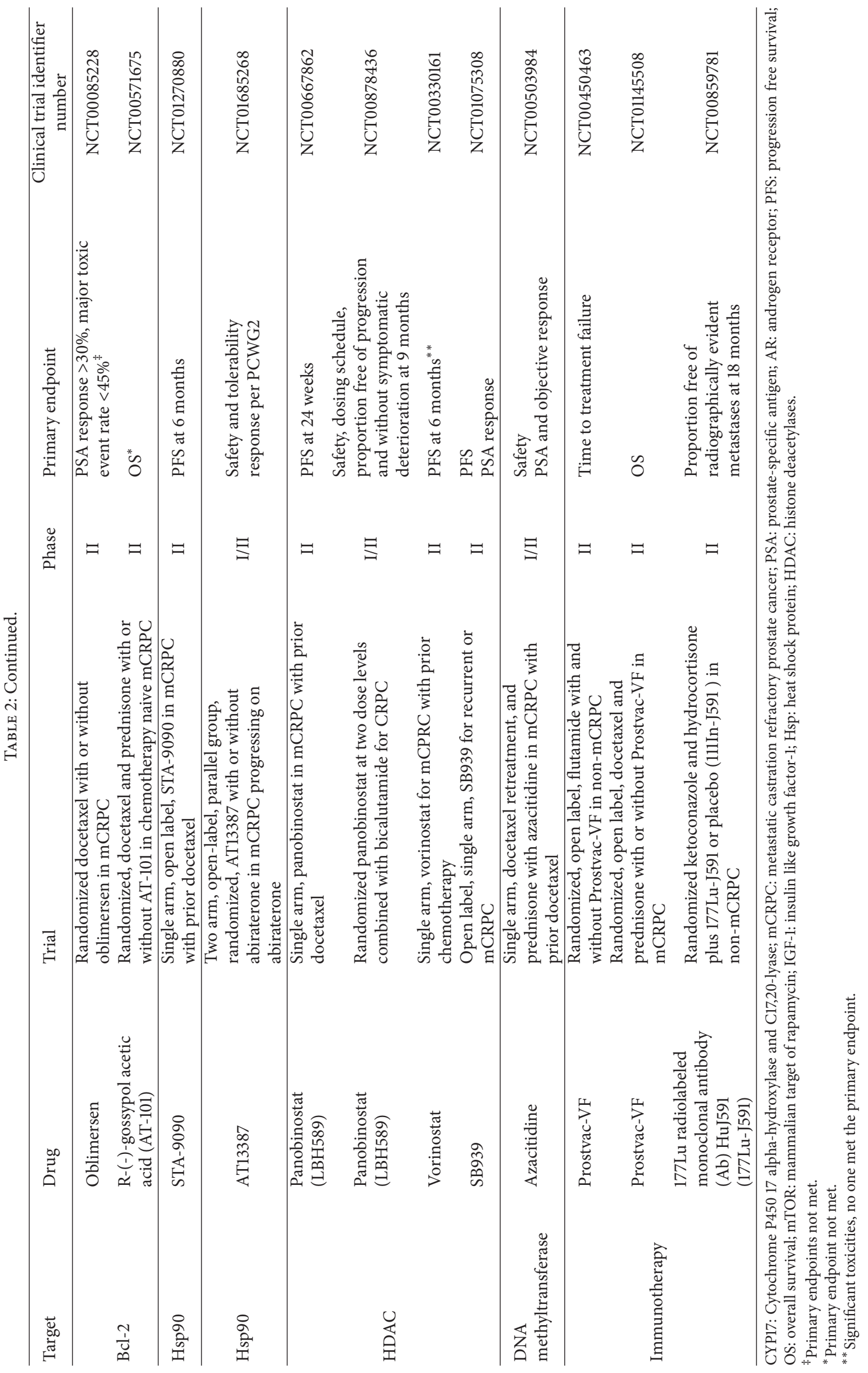


III trial of men with CRPC with prior docetaxel therapy, enzalutamide when compared to placebo, significantly improved overall survival (18.4 months versus 13.6 months; $P<0.001$ ), leading to its regulatory approval in this setting [12]. Another phase III trial of enzalutamide in chemotherapy naive men with CPRC is currently ongoing (Table 1 ).

ARN-509 is a novel small molecule AR antagonist, is structurally and mechanistically similar to enzalutamide, and impairs AR nuclear translocation, as well as AR binding to DNA. In phase I/II study of men with metastatic CRPC, ARN-509 was well tolerated and showed pharmacodynamic evidence of $\mathrm{AR}$ antagonism with promising clinical activity. Phase II dose was determined to be $240 \mathrm{mg}$ per day. The most common treatment related grade 1-2 side effects were fatigue, nausea, and pain [13] (Table 2). The phase II portion of the study included three distinct population of men with CRPC: nonmetastatic treatment-naive CRPC, metastatic CRPC (treatment naïve), and metastatic CRPC (with prior treatment with abiraterone acetate). The primary endpoint was PSA response rate at 12 weeks according to the Prostate Cancer Working Group 2 Criteria. Preliminary results were reported at the 2013 genitourinary cancer symposium. Among 47 men with non-metastatic CRPC, the 12-week PSA response was $91 \%$, and the time to PSA progression had not been reached [14]. Among 46 men with metastatic CRPC, 26 were treatment naïve, and 21 had prior treatment with abiraterone acetate. At 12 weeks, the PSA response was $88 \%$ in the treatment naïve cohort. Notably, 29\% men with prior treatment with abiraterone acetate had PSA response after 12 weeks, thus indicating activity of ARN-509 in the subset of men with CRPC that developed resistance to abiraterone acetate [15].

Other promising rationally designed drugs inhibiting AR signaling include TOK-001 (galeterone), EZN-4176, and EPI-001. TOK-001, formerly known as VN/124-1, inhibits prostate cancer growth by multiple mechanisms. In addition to inhibiting CYP17, it directly antagonizes the AR receptor and also downregulates AR protein expression [16]. A phase I/II trial of TOK-001 has been initiated in chemonaive patients with mCRPC (Table 2). EZN-4176, an AR mRNA antagonist, is a locked nucleic acid (LNA) oligonucleotide that downregulates $\mathrm{AR}$ mRNA and is currently ongoing development in a phase I trial of men with CRPC [17]. EPI001 inhibits the $\mathrm{N}$ terminal domain of the AR, which confers transcriptional activity, and has the capability to overcome castration resistance associated with a gain of function mutations of the ligand binding domain, and expression of constitutionally active splice variants of the AR. EPI-001 is currently awaiting clinical trial development in [18].

\section{Targeting Androgen Independent Molecular Pathways Implicated in Stromal-Epithelial Crosstalk and in Shaping Tumor Microenvironment}

3.1. Targeting Src Kinase Signaling. The Src family of nonreceptor protein tyrosine kinases (SFKs) is upregulated in various human malignancies, including prostate cancer and their expression directly correlates with disease progression and metastasis $[19,20]$. In addition, Src signaling plays an important role in normal bone turnover, and is essential for normal osteoclast functioning, as well as osteoblast proliferation, and has been implicated in the promotion of bone metastasis in prostate cancer [20].

Dasatinib (BMS-354825) is a selective small molecule inhibitor of SFKs and other tyrosine kinases, including Bcr$\mathrm{Abl}, \mathrm{Kit}$, and PDGFR $\beta$. A recent phase II study of chemotherapy naïve patients with CRPC has shown promising results. Forty-three percent of patients at week 12 and 19\% patients at week 24 had radiographically stable disease. Additionally, at week $12,51 \%$ of patients achieved $\geq 40 \%$ reduction in urinary N-telopeptide, while $60 \%$ had reduction in bone alkaline phosphatase. Treatment with dasatinib was generally well tolerated. Based on the positive results from a separate phase I/II study, which combined dasatinib with docetaxel, a randomized phase III study comparing docetaxel with dasatinib to docetaxel with placebo in castration resistant prostate cancer was conducted. The results, however, failed to show an improvement in overall survival [21]. Additionally, dasatinib is being tested in two randomized phase II trials in combination with abiraterone and cediranib (VEGF TKI), respectively (Table 2).

Saracatinib (AZD0530) is an oral non-receptor tyrosine kinase inhibitor targeting Src kinases and has been shown to have activity in orthotopic animal models of CRPC [22]. Two phase II studies of saracatinib in CRPC in chemotherapy naive and postdocetaxel settings, respectively, are underway (Table 1).

3.2. Targeting PI3K/Akt/mTOR Pathway. The phosphatidylinositol 3-kinase (PI3 K)/Akt/mammalian target of rapamycin (mTOR) signaling pathway regulates multiple physiological cell processes, which include metabolism, proliferation, differentiation, survival, migration, and angiogenesis. The lipid and protein phosphatase, PTEN (phosphatase and tensin homology protein), is a key negative regulator of Akt activity. Aberrant expression of PI3K and AKT1 genes or loss of PTEN tumor suppression gene leads to downstream upregulation of mTOR and tumorigenesis. Although, everolimus does not have clear significant single agent activity in CRPC, it holds promise in combination therapy with chemotherapeutics given the fact that it restores sensitivity to chemotherapy as do other drugs within the class [23]. Preliminary results of a phase I/II trial combining docetaxel and temsirolimus have showed acceptable safety and antitumor activity and a phase II expansion cohort is underway [24]. Furthermore, multiple phase II studies of mTOR inhibitors as single agent or in combination with chemotherapeutics or biologic agents are ongoing (Table 2).

\subsection{Targeting Vascular Endothelial Growth Factor (VEGF)} Pathway. Angiogenesis and neovascularization are necessary for the growth and metastasis of solid tumors. Vascular endothelial growth factor (VEGF) plays a pivotal role in angiogenesis and neovascularization. There is clinical evidence that the simultaneous use of angiogenesis inhibitors 
and chemotherapeutic drugs may lead to improved outcomes in patients with CRPC [25-27]. Multiple agents have been tested in phase III trials of men with metastatic CRPC.

Bevacizumab is a humanized monoclonal antibody that inhibits angiogenesis by neutralizing circulating vascular endothelial growth factor (VEGF). Sunitinib is a tyrosine kinase inhibitor (TKI) which similarly targets VEGF receptor. Aflibercept (VEGF Trap) is a recombinant humanized fusion protein consisting of the VEGF extracellular domains and the Fc portion of human immunoglobulin IgG1. However, none of these agents have shown overall survival advantage in phase III trials of men with metastatic CRPC (Table 1).

3.4. Targeting Insulin Like Growth Factor (IGF) Pathway. The insulin like growth factor (IGF) pathway, which includes IGF receptor-1 (IGF-1R) and its ligands, IGF-I and IGF-II, not only plays a major role in growth, development, and maintenance of homeostasis in normal cells, but also the proliferation of cancers cells, including prostate cancer cells [28]. Higher levels of circulating IGF-I correlates with increased risk of developing prostate cancer, as well as metastatic disease. Blockade of IGF-IR in combination with chemotherapy leads to chemosensitization in androgen-independent prostate cancer cell lines and improved docetaxel antitumor activity in animal models [28].

Figitumumab and cixutumumab are fully human monoclonal antibodies targeting IGF-1R. In a phase Ib trial, a combination of figitumumab and docetaxel was well tolerated in patients with a variety of solid tumors, with three patients with CRPC demonstrating an objective response [28]. Encouraged by these results, a phase II study of this combination has been initiated in the setting of CRPC (Table 2). In a phase II study, chemotherapy naïve men with metastatic CRPC were treated with cixutumumab at two different dosing schemes in both cohorts. Approximately $30 \%$ of patients had disease stabilization for $\geq 6$ months [29]. The most common drug-related adverse events were fatigue and asymptomatic hyperglycemia. Phase II studies of cixutumumab, either as monotherapy or in combination with temsirolimus, are ongoing in men with chemotherapy naïve metastatic CRPC (Table 2).

3.5. Targeting Bcl-2. Overexpression of $\mathrm{Bcl}-2$ is observed in a significant number of men with CRPC. It plays a key role in the onset of castration refractoriness and contributes to the resistance to radiation and docetaxel therapy [30]. Inhibition of $\mathrm{Bcl}-2$ expression leads to increased apoptosis, as well as diminished proliferation and angiogenesis [31].

Oblimersen (Genasense, G3139) is an 18-base synthetic oligonucleotide strand that hybridizes with the first six codons of the Bcl-2 RNA transcript, resulting in degradation by endogenous RNase $\mathrm{H}$ and the inhibition of $\mathrm{Bcl}-2$ protein expression [31]. In a multicenter, phase II study men with chemotherapy naïve metastatic CRPC with PSA progression, patients were randomized to receive docetaxel with or without oblimersen [30]. Oblimersen was given on days 1-5 to achieve downregulation of $\mathrm{Bcl}-2$ prior to docetaxel, which was given on day 5. Oblimersen was then continued for two more days after docetaxel to allow for adequate coexposure of the two drugs. However, the primary endpoints of the study (confirmed PSA response rate of $>30 \%$ and a major toxic event rate $<45 \%$ in the combination arm) were not met [30].

AT-101 (R-gossypol acetic acid) is a polyphenolic compound derived from the cottonseed plant [32]. Acting as a BH3 mimetic, AT-101 inhibits the function of Bcl-2 family member proteins by preventing their binding with proapoptotic proteins and upregulates proapoptotic factors. In a phase II study, men with progressive metastatic CRPC received docetaxel and prednisone with or without AT-101 [33]. Although, overall survival, the primary endpoint, was not improved, a potential benefit was observed in a subgroup of high-risk patients.

These results suggest that apoptotic failure may result from molecular mechanisms other than Bcl-2 over expression. Men with documented underlying Bcl-2 over expression, as a mechanism of chemoresistance, may be more appropriate candidates for combinatorial regimen employing Bcl-2 targeted therapies.

3.6. Targeting Cytoprotective Chaperone Proteins. Clusterin is a cytoprotective chaperone whose transcription is promoted by the androgen receptor and by heat shock factor-1, a key mediator of carcinogenesis [34]. Over expression of clusterin in prostate cancer has been correlated with progression to CRPC $[34,35]$.

Custirsen (OGX-011) is an antisense oligonucleotide to the clusterin mRNA translation initiation site that potently inhibits clusterin expression and enhances the efficacy of anticancer therapies in vitro and in vivo. In a phase II study, men with metastatic CRPC were treated with docetaxel and prednisone with or without OGX-011. Although, there was no improvement in PSA response (the primary endpoint), overall survival (secondary endpoint) was improved in the OGX-011 arm (23.8 months versus 16.9 months) [34]. Currently, three phase III trials combing OGX-011 with chemotherapeutic agents are ongoing in metastatic CRPC (Table 1).

HSP-90 is another multifaceted molecular chaperone implicated in the progression of prostate cancer by the induction of several upstream signaling pathways, which promote aberrant androgen receptor activation and stabilize the androgen receptor protein. Phase II trials are currently ongoing in metastatic CRPC using HSP-90 inhibitors (Table 2) [36].

3.7. Targeting Hepatocyte Growth Factor/c-Met Signaling Pathway. Hepatocyte growth factor (HGF) is a potent oncogenic protein, which often acts synergistically with VEGF on endothelial cells, as well as several other cellular signaling pathways, including Ras/MEK pathway and PI3K/AKT pathway [37]. HGF acts through its receptor HGFR, also known as c-Met. The HGF/c-Met pathway is overexpressed in prostate cancer and promotes metastasis. Androgen receptor suppresses c-Met transcription, and c-Met expression is up regulated by castration [38]. 
Cabozantinib (XL-184) is an inhibitor of c-MET and VEGF receptor tyrosine kinases. In a phase II study of men with metastatic CRPC, treatment with cabozantinib, when compared to placebo, resulted in improved PFS, reduction of soft tissue lesions, resolution of bone scans, and decreased pain [39]. Currently, two phase III trials of cabozantinib are ongoing in men with metastatic CRPC, with prior docetaxel and with prior abiraterone or MDV3100 (Table 1).

3.8. Targeting Epigenetic Pathways. AR, a transcription factor, binds with the androgen response elements (AREs) located throughout the genome and recruits various cofactors, such as histone acetyltransferases (HATs) and histone deacetylases (HDACs). This leads to the formation of multiprotein complexes involved in the AR mediated transcriptional regulation of various genes implicated in prostate cancer growth and proliferation [40]. In prostate cancer, HDAC inhibition leads to decreased proliferation of cell lines and decreased tumor growth in preclinical models [41].

Vorinostat is a small molecule inhibitor of class I and II HDACs and has shown promising antitumor activity in prostate cancer cell lines and animal models of prostate cancer. Unfortunately, a phase II trial in patients with mCRPC showed no PSA or objective response and an unacceptable toxicity profile. Panobinostat, a pan-deacetylase inhibitor, has undergone testing in a phase I trial in combination with docetaxel in CRPC [42]. Furthermore, panobinostat is being tested in a phase I/II study in combination with bicalutamide and in a phase II study, as monotherapy for patients with CRPC (Table 2).

Another epigenetic pathway of interest is DNA methylation. An open chromatin structure induced by hypomethylation facilitates gene transcription, whereas a closed structure inhibits transcription. Azacitidine, an inhibitor of DNA methyltransferase, induces hypomethylation and reverses the silencing of tumor suppressor genes. It has been shown to restore sensitivity to androgen deprivation therapy, as well as chemotherapy in preclinical models of prostate cancer [23]. In a phase II trial of men with chemonaive CRPC progressing on combined androgen blockade, addition of azacitidine significantly prolonged PSA doubling time [43]. Currently, azacitidine is being tested in a phase II trial in combination with docetaxel in CRPC with prior docetaxel therapy (Table 2).

3.9. Targeting Bone Metastasis. The majority of patients with mCRPC develop osteoblastic bone metastasis accompanied by simultaneous bone destruction, due to increased osteoclastic activity. The receptor activator of nuclear factor- $\mathrm{kB}$ ligand (RANKL) is critical for the formation, function, and survival of osteoclasts. In preclinical models of prostate cancer, inhibition of osteoclasts leads to improvement of sclerotic changes in the bones [44]. In a phase III trial, zoledronic acid, an inhibitor of osteoclasts, significantly decreased the incidence of skeletal related events (SRE) and increased the median time to the first SRE, over placebo [45].

Denosumab is a monoclonal antibody against RANKL. Compared to zoledronic acid, denosumab improved median time to first SRE in a phase III trial of men with CRPC with bone metastasis (20.7 versus 17.1 months; HR: 0.82; 95\% confidence interval, $0.71-0.95 ; P=0.0002$ for noninferiority; $P=0.008$ for superiority).

Radium-223 chloride ( $\mathrm{Ra}-223)$, a radiopharmaceutical agent, targets bone metastasis by emitting high energy alphaparticles of extremely short range $(<100 \mu \mathrm{m})$. In a phase III, placebo controlled trial of men with symptomatic CRPC with bone metastasis, Ra-223 significantly improved overall survival (14.0 versus 11.2 months, two-sided $P=0.00185$; $\mathrm{HR}=$ $0.695 ; 95 \%$ CI, 0.552-0.875) and delayed time to first SRE (13.6 versus 8.4 months, $P=0.00046$; $\mathrm{HR}=0.610$; $95 \% \mathrm{CI}, 0.461-$ $0.807)$ [46].

3.10. Modulating the Immune System. Regulatory approval of sipuleucel-T for mCRPC has validated modulation of the immune system as an effective strategy in prostate cancer. Sipuleucel-T consists of autologous antigen presenting cells enriched for a CD54+ DC fraction that are harvested by leukopheresis and cultured with a fusion protein (PA2024), comprising of prostate acid phosphatase (PAP) and granulocyte-monocyte colony stimulating factor (GMCSF) $[47,48]$. In a phase III trial of men with asymptomatic chemonaive metastatic CRPC, the median overall survival was significantly improved with sipuleucel-T over placebo (25.8 versus 21.7 months; $\mathrm{HR}=0.77 ; P=0.02$ ), with a relative reduction of $22 \%$ in the risk of death in the sipuleucel-T group ( $\mathrm{HR}=0.78 ; P=0.03$ ) [48]. Upon disease progression, $64 \%$ men in the control arm crossed over to a nonrandomized open-label protocol to receive an investigational autologous immunotherapy made from cryopreserved cells (APC8015F). In an exploratory analysis, after adjusting for the effect of APC8015F and assuming that APC8015F was as effective as sipuleucel-T, the estimated overall benefit with sipuleucel-T was 7.8 months [49].

3.11. Virus-Based Vaccines. The inherent immunogenicity of viruses and the high level of gene expression seen with viral vectors lead to a strong inflammatory response and may lead to an improved immune response against tumor antigens expressed by viruses [50]. Prostvac-VF utilizes a heterologous prime/boost vaccination strategy. It consists of two recombinant viral vectors (vaccinia vector and fowlpox vector), each encoding transgenes for PSA and TRICOM. TRICOM consists of costimulatory molecules, including ICAM (intercellular addition molecules)-1 (CD54), B7.1 (CD80), and leukocyte function-associated antigen-3 (LFA-3)(CD58) [51]. In a randomized phase II trial of men with chemonaive, minimally symptomatic metastatic CRPC, ProstvacVF extended overall survival by 8.5 months over placebo (25.1 versus 16.6 months, $P=0.0061 ; \mathrm{HR}=0.56 ; 95 \%$ CI, 0.37-0.85) [52]. Ongoing clinical trials of Prostvac-VF in advanced phases include those in the setting of non-mCRPC, in asymptomatic/minimally symptomatic metastatic CRPC and in symptomatic metastatic CRPC (in combination with docetaxel) (Tables 1 and 2).

3.12. Cytotoxic T Lymphocyte Antigen 4 (CTLA-4) Antibody. Cytotoxic T lymphocyte antigen 4 (CTLA-4) is a key negative 
regulator of T-cell responses, inhibits recognition of selfantigens by $\mathrm{T}$ cells, and can downregulate the antitumor immune response. Ipilimumab and tremelimumab are fully human, monoclonal antibodies against CTLA-4. Objective and PSA responses have been described in phase II studies of ipilimumab in prostate cancer $[53,54]$. Recently, two phase III trials of ipilimumab in chemonaive- and postdocetaxel metastatic CRPC settings have completed accrual with results expected in the near future (Table 1).

3.13. Radiolabeled Monoclonal Antibody. Monoclonal antibodies (MoAbs) targeting prostate surface membrane antigen (PSMA) are in advanced phases of development. PSMA is a type II membrane glycoprotein, which is markedly upregulated in prostate cancer [55]. Deimmunized murine MoAb J591 (muJ591) has been chosen as a vehicle to deliver radioisotopes, because of its high affinity for PSMA [56]. Among various radioisotopes used with mu J591, 177 lutetium can be administered in higher doses, with comparatively less radiation to the marrow and because of its gamma emission, it enables imaging to be performed using the treatment doses [55]. A randomized, phase II study is currently evaluating efficacy of 177Lu-J591 (versus placebo, i.e., 111 In-J591) in combination with ketoconazole and hydrocortisone in nonmetastatic CRPC (Table 2).

3.14. Tasquinimod. Tasquinimod is a quinoline-3-carboxamide analog that possesses antiangiogenic and immunomodulatory properties [57]. It significantly delayed disease progression, compared to placebo, in a randomized phase II trial of minimally symptomatic men with metastatic CRPC. A phase III, placebo controlled, randomized trial of tasquinimod has recently completed accrual with the primary endpoint of progression free survival (Table 1).

\section{Conclusions}

Herein, we have provided a thorough, but selective list of androgen dependent and independent molecular pathways recognized to drive the progression of CRPC. Additionally, we summarized the novel agents targeting these pathways, which have been recently approved or have reached advanced stages of clinical development. With the discovery of increased intratumoral expression of key enzymes in the steroid synthesis pathway, agents such as abiraterone acetate, orteronel, and TOK-001 were developed to further minimize androgen-driven tumor progression. When AR gene amplification and mutations were shown by in situ studies, enzalutamide and ARN-509 were developed to abrogate the actions of AR in facilitating tumor progression. Regulatory approval of sipuleucel-T for minimally symptomatic mCRPC has revolutionized the way immune therapy is used in management of malignancy in general. Development of other promising immune-modulatory agents such as Prostvac-VF, ipilimumab, and tasquinimod have reached phase III trials. Classic pathways common to many malignancies, such as apoptotic pathways (bcl2), src-kinase, p53, and PTEN, are also dysregulated by mutations, gene duplications/deletions, and chromosomal changes. These discoveries have provided the rationale for testing agents targeting these pathways for treatment of CRPC.

The recent proliferation of therapeutic agents for treatment of metastatic CRPC is indicative of the dramatic changes occurring in the field. Amidst this changing landscape of treatment options for metastatic CRPC, many ongoing trials are likely to result in regulatory approval of newer agents. Advances in evidenced based oncology have led to cost-efficient deep sequencing and individual genome sequencing capability. These and similar tools will give physicians insight into the pathophysiology of the tumors of each individual and help personalize treatment based on the underlying molecular pathway or pathways, driving tumor progression. More rationally designed studies based on predictive biomarkers are needed to guide our way through the changing landscape of treatment and the ever increasing treatment options.

\section{Authors' Contribution}

All authors participated in concept and design, collection of data, writing of the paper, and approval of the paper. Jesal C. Patel and Benjamin L. Maughan are equal contributors to the paper.

\section{Acknowledgment}

Neeraj Agarwal has received honorarium from Dendreon Inc, Seattle, WA, USA.

\section{References}

[1] R. Siegel, D. Naishadham, and A. Jemal, "Cancer statistics," A Cancer Journal for Clinicians, vol. 63, no. 1, pp. 11-30, 2013.

[2] Y. Chen, N. J. Clegg, and H. I. Scher, "Anti-androgens and androgen-depleting therapies in prostate cancer: new agents for an established target," The Lancet Oncology, vol. 10, no. 10, pp. 981-991, 2009.

[3] A. H. M. Reid, G. Attard, E. Barrie, and J. S. de Bono, "CYP17 inhibition as a hormonal strategy for prostate cancer," Nature Clinical Practice Urology, vol. 5, no. 11, pp. 610-620, 2008.

[4] J. Edwards, N. S. Krishna, K. M. Grigor, and J. M. S. Bartlett, "Androgen receptor gene amplification and protein expression in hormone refractory prostate cancer," British Journal of Cancer, vol. 89, no. 3, pp. 552-556, 2003.

[5] M. P. Steinkamp, O. A. O’Mahony, M. Brogley et al., "Treatmentdependent androgen receptor mutations in prostate cancer exploit multiple mechanisms to evade therapy," Cancer Research, vol. 69, no. 10, pp. 4434-4442, 2009.

[6] N. Agarwal, T. E. Hutson, N. J. Vogelzang, and G. Sonpavde, "Abiraterone acetate: a promising drug for the treatment of castration-resistant prostate cancer," Future Oncology, vol. 6, no. 5, pp. 665-679, 2010.

[7] J. S. de Bono, C. J. Logothetis, A. Molina et al., "Abiraterone and increased survival in metastatic prostate cancer.," The New England Journal of Medicine, vol. 364, no. 21, pp. 1995-2005, 2011.

[8] C. J. Ryan, M. R. Smith, J. S. de Bono et al., "Abiraterone in metastatic prostate cancer without previous chemotherapy," The 
New England Journal of Medicine, vol. 368, no. 2, pp. 138-148, 2013.

[9] D. B. Agus, W. M. Stadler, D. H. Shevrin et al., "Efficacy, and pharmacodynamics of the investigational agent orteronel (TAK-700) in metastatic castration-resistant prostate cancer (mCRPC): updated data from a phase I/II study," Journal of Clinical Oncology, vol. 30, no. 5, supplement 98, 2012.

[10] M. Hussain, P. Corn, D. Michaelson et al., "Activity and safety of the investigational agent orteronel in men with nonmetastatic castration-resistant prostate cancer and rising prostate-specific antigen: results of a phase 2 study," in Proceedings of the 27th Annual European Association of Urology, pp. 24-28, Paris, France, February 2012.

[11] C. Tran, "Development of a second-generation antiandrogen for treatment of advanced prostate cancer," Science, vol. 324, no. 5928, pp. 787-790, 2009.

[12] H. I. Scher, K. Fizazi, F. Saad et al., "Increased survival with enzalutamide in prostate cancer after chemotherapy," The New England Journal of Medicine, vol. 367, no. 13, pp. 1187-1197, 2012.

[13] D. E. Rathkopf, D. C. Danila, M. J. Morris et al., "Phase I/II safety and pharmacokinetic (PK) study of ARN-509 in patients with metastatic castration-resistant prostate cancer (mCRPC): phase I results of a prostate cancer clinical trials consortium study," Journal of Clinical Oncology, vol. 30, 5, 2012.

[14] M. R. Smith, E. S. Antonarakis, C. J. Ryan et al., "ARN-509 in men with high-risk nonmetastatic castration-resistant prostate cancer (CRPC)," ASCO Meeting Abstracts, vol. 31, 6, 2013.

[15] D. E. Rathkopf, E. S. Antonarakis, N. D. Shore et al., "ARN509 in men with metastatic castration-resistant prostate cancer (mCRPC)," ASCO Meeting Abstracts, vol. 31, 6, 2013.

[16] T. Vasaitis, A. Belosay, A. Schayowitz et al., "Androgen receptor inactivation contributes to antitumor efficacy of $17 \alpha$-hydroxylase/17,20-lyase inhibitor $3 \beta$-hydroxy-17-(1H-benzimidazole-1-yl)androsta-5,16-diene in prostate cancer," Molecular Cancer Therapeutics, vol. 7, no. 8, pp. 2348-2357, 2008.

[17] Y. Zhang, S. Castaneda, M. Dumble et al., "Reduced expression of the androgen receptor by third generation of antisense shows antitumor activity in models of prostate cancer," Molecular Cancer Therapeutics, vol. 10, no. 12, pp. 2309-2319, 2011.

[18] R. J. Andersen, N. R. Mawji, J. Wang et al., "Regression of castrate-recurrent prostate cancer by a small-molecule inhibitor of the amino-terminus domain of the androgen receptor," Cancer Cell, vol. 17, no. 6, pp. 535-546, 2010.

[19] J. M. Summy and G. E. Gallick, "Src family kinases in tumor progression and metastasis," Cancer and Metastasis Reviews, vol. 22, no. 4, pp. 337-358, 2003.

[20] K. Fizazi, "The role of Src in prostate cancer," Annals of Oncology, vol. 18, no. 11, pp. 1765-1773, 2007.

[21] J. C. Araujo, G. C. Trudel, F. Saad et al., "Overall survival (OS) and safety of dasatinib/docetaxel versus docetaxel in patients with metastatic castration-resistant prostate cancer (mCRPC): results from the randomized phase III READY trial," ASCO Meeting Abstracts, vol. 31, 6, 2013.

[22] M. Karlou, V. Tzelepi, and E. Efstathiou, "Therapeutic targeting of the prostate cancer microenvironment," Nature Reviews Urology, vol. 7, no. 9, pp. 494-509, 2010.

[23] E. S. Antonarakis, M. A. Carducci, and M. A. Eisenberger, "Novel targeted therapeutics for metastatic castration-resistant prostate cancer," Cancer Letters, vol. 291, no. 1, pp. 1-13, 2010.

[24] I. Duran, C. Montagut, E. Calvo et al., "Overcoming docetaxel resistance in advanced castration-resistant prostate cancer
(CRPC): a phase I/II trial of the combination of temsirolimus and docetaxel," Journal of Clinical Oncology, vol. 30, 5, 2012.

[25] W. D. Figg, P. Arlen, J. Gulley et al., "A randomized phase II trial of docetaxel (taxotere) plus thalidomide in androgenindependent prostate cancer," Seminars in Oncology, vol. 28, no. 4, pp. 62-66, 2001.

[26] W. L. Dahut, J. L. Gulley, P. M. Arlen et al., "Randomized phase II trial of docetaxel plus thalidomide in androgen-independent prostate cancer," Journal of Clinical Oncology, vol. 22, no. 13, pp. 2532-2539, 2004.

[27] W. D. Figg, H. Li, T. Sissung et al., "Pre-clinical and clinical evaluation of estramustine, docetaxel and thalidomide combination in androgen-independent prostate cancer," BJU International, vol. 99, no. 5, pp. 1047-1055, 2007.

[28] L. R. Molife, P. C. Fong, L. Paccagnella et al., "The insulin-like growth factor-I receptor inhibitor figitumumab (CP-751,871) in combination with docetaxel in patients with advanced solid tumours: results of a phase $\mathrm{Ib}$ dose-escalation, open-label study," British Journal of Cancer, vol. 103, no. 3, pp. 332-339, 2010.

[29] C. S. Higano, J. J. Alumkal, C. J. Ryan et al., "A phase II study of cixutumumab (IMC-A12), a monoclonal antibody (MAb) against the insulin-like growth factor 1 receptor (IGF-IR), monotherapy in metastatic castration-resistant prostate cancer (mCRPC): feasibility of every 3-week dosing and updated results," Genitourinary Cancers Symposium, vol. 189, 2010.

[30] C. N. Sternberg, H. Dumez, H. Van Poppel et al., "Docetaxel plus oblimersen sodium (Bcl-2 antisense oligonucleotide): an EORTC multicenter, randomized phase II study in patients with castration-resistant prostate cancer," Annals of Oncology, vol. 20, no. 7, pp. 1264-1269, 2009.

[31] S. Anai, S. Goodison, K. Shiverick, Y. Hirao, B. D. Brown, and C. J. Rosser, "Knock-down of Bcl-2 by antisense oligodeoxynucleotides induces radiosensitization and inhibition of angiogenesis in human PC-3 prostate tumor xenografts," Molecular Cancer Therapeutics, vol. 6, no. 1, pp. 101-111, 2007.

[32] G. Liu, W. K. Kelly, G. Wilding, L. Leopold, K. Brill, and B. Somer, "An open-label, multicenter, phase I/II study of singleagent AT-101 in men with castrate-resistant prostate cancer," Clinical Cancer Research, vol. 15, no. 9, pp. 3172-3176, 2009.

[33] G. Sonpavde, V. Matveev, J. M. Burke et al., "Randomized phase II trial of docetaxel plus prednisone in combination with placebo or AT-101, an oral small molecule Bcl-2 family antagonist, as first-line therapy for metastatic castration-resistant prostate cancer," Annals of Oncology, vol. 23, no. 7, pp. 1803$1808,2012$.

[34] K. N. Chi, S. J. Hotte, E. Y. Yu et al., "Randomized phase II study of docetaxel and prednisone with or without OGX-011 in patients with metastatic castration-resistant prostate cancer," Journal of Clinical Oncology, vol. 28, no. 27, pp. 4247-4254, 2010.

[35] A. Zoubeidi, K. Chi, and M. Gleave, "Targeting the cytoprotective chaperone, clusterin, for treatment of advanced cancer," Clinical Cancer Research, vol. 16, no. 4, pp. 1088-1093, 2010.

[36] F. Stavridi, E. M. Karapanagiotou, and K. N. Syrigos, “Targeted therapeutic approaches for hormone-refractory prostate cancer," Cancer Treatment Reviews, vol. 36, no. 2, pp. 122-130, 2010.

[37] W. K. You and D. M. M, “The hepatocyte growth factor/c-met signaling pathway as a therapeutic target to inhibit angiogenesis," Journal of Biochemistry and Molecular Biology, vol. 41, no. 12, pp. 833-839, 2008. 
[38] W. H. Tu, C. Zhu, C. Clark, J. G. Christensen, and Z. Sun, "Efficacy of c-Met inhibitor for advanced prostate cancer," BMC Cancer, vol. 10, article 556, 2010.

[39] D. C. Smith, M. R. Smith, C. Sweeney et al., "Cabozantinib in patients with advanced prostate cancer: results of a phase II randomized discontinuation trial," Journal of clinical oncology, vol. 31, no. 4, pp. 412-419, 2012.

[40] D. N. Lavery and C. L. Bevan, "Androgen receptor signalling in prostate cancer: the functional consequences of acetylation," Journal of Biomedicine and Biotechnology, vol. 2011, Article ID 862125, 7 pages, 2011.

[41] D. Bradley, D. Rathkopf, R. Dunn et al., "Vorinostat in advanced prostate cancer patients progressing on prior chemotherapy (National Cancer Institute Trial 6862): trial results and interleukin- 6 analysis: a study by the department of defense prostate cancer clinical Trial consortium and university of chicago phase 2 consortium," Cancer, vol. 115, no. 23, pp. 55415549, 2009.

[42] D. Rathkopf, B. Y. Wong, R. W. Ross et al., "A phase I study of oral panobinostat alone and in combination with docetaxel in patients with castration-resistant prostate cancer," Cancer Chemotherapy and Pharmacology, vol. 66, no. 1, pp. 181-189, 2010.

[43] G. Sonpavde, A. M. Aparicio, F. Zhan et al., "Azacitidine favorably modulates PSA kinetics correlating with plasma DNA LINE-1 hypomethylation in men with chemonaive castrationresistant prostate cancer," Urologic Oncology, vol. 29, no. 6, pp. 682-689, 2011.

[44] K. Fizazi, M. Carducci, M. Smith et al., "Denosumab versus zoledronic acid for treatment of bone metastases in men with castration-resistant prostate cancer: a randomised, doubleblind study," The Lancet, vol. 377, no. 9768, pp. 813-822, 2011.

[45] F. Saad, D. M. Gleason, R. Murray et al., "Long-term efficacy of zoledronic acid for the prevention of skeletal complications in patients with metastatic hormone-refractory prostate cancer," Journal of the National Cancer Institute, vol. 96, no. 11, pp. 879882, 2004.

[46] A. O. Sartor, D. Heinrich, S. I. Helle et al. et al., "Radium223 chloride impact on skeletal-related events in patients with castration-resistant prostate cancer (CRPC) with bone metastases: a phase III randomized trial (ALSYMPCA)," Journal of Clinical Oncology, vol. 30, 5, 2012.

[47] N. Agarwal, N. Vogelzang, and N. J, Development of Novel Immune Interventions For Genito-Urinary Cancers. Cancer Vaccines, Adrian Bot, Mihail Obrocea \& Francesco Marincola, Informa Healthcare, London, UK, 2011.

[48] P. W. Kantoff, C. S. Higano, N. D. Shore et al., "SipuleucelT immunotherapy for castration-resistant prostate cancer," The New England Journal of Medicine, vol. 363, no. 5, pp. 411-422, 2010.

[49] L. G. Gomella, C. Nabhan, T. DeVries, J. B. Whitmore, M. W. Frohlich, and D. J. George, "Estimating the overall survival benefit of sipuleucel-T in the IMPACT trial accounting for crossover treatment in control subjects with autologous immunotherapy generated from cryopreserved cells," in American Urological Association Annual Meeting (AUA '12), 2012.

[50] J. Schlom, M. Vergati, C. Intrivici, N. Y. Huen, and K. Y. Tsang, "Strategies for cancer vaccine development," Journal of Biomedicine and Biotechnology, vol. 2010, Article ID 596432, 13 pages, 2010.
[51] G. Sonpavde, K. M. Slawin, D. M. Spencer, and J. M. Levitt, "Emerging vaccine therapy approaches for prostate cancer," Reviews in Urology, vol. 12, no. 1, pp. 25-34, 2010.

[52] P. W. Kantoff, T. J. Schuetz, B. A. Blumenstein et al., “Overall survival analysis of a phase II randomized controlled trial of a poxviral-based PSA-targeted immunotherapy in metastatic castration-resistant prostate cancer," Journal of Clinical Oncology, vol. 28, no. 7, pp. 1099-1105, 2010.

[53] L. F. Langer, T. M. Clay, and M. A. Morse, "Update on anti-CTLA-4 antibodies in clinical trials," Expert Opinion on Biological Therapy, vol. 7, no. 8, pp. 1245-1256, 2007.

[54] D. O’Mahony, J. C. Morris, C. Quinn et al., “A pilot study of CTLA-4 blockade after cancer vaccine failure in patients with advanced malignancy," Clinical Cancer Research, vol. 13, no. 3, pp. 958-964, 2007.

[55] S. T. Tagawa, H. Beltran, S. Vallabhajosula et al., "Anti-prostatespecific membrane antigen-based radioimmunotherapy for prostate cancer," Cancer, vol. 116, no. 4, pp. 1075-1083, 2010.

[56] N. H. Bander, D. M. Nanus, M. I. Milowsky, L. Kostakoglu, S. Vallabahajosula, and S. J. Goldsmith, "Targeted systemic therapy of prostate cancer with a monoclonal antibody to prostate-specific membrane antigen," Seminars in Oncology, vol. 30, no. 5, pp. 667-677, 2003.

[57] J. T. Isaacs, "The long and winding road for the development of tasquinimod as an oral second-generation quinoline-3carboxamide antiangiogenic drug for the treatment of prostate cancer," Expert Opinion on Investigational Drugs, vol. 19, no. 10, pp. 1235-1243, 2010. 


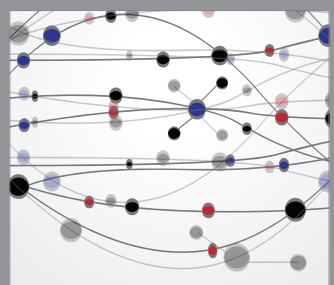

The Scientific World Journal
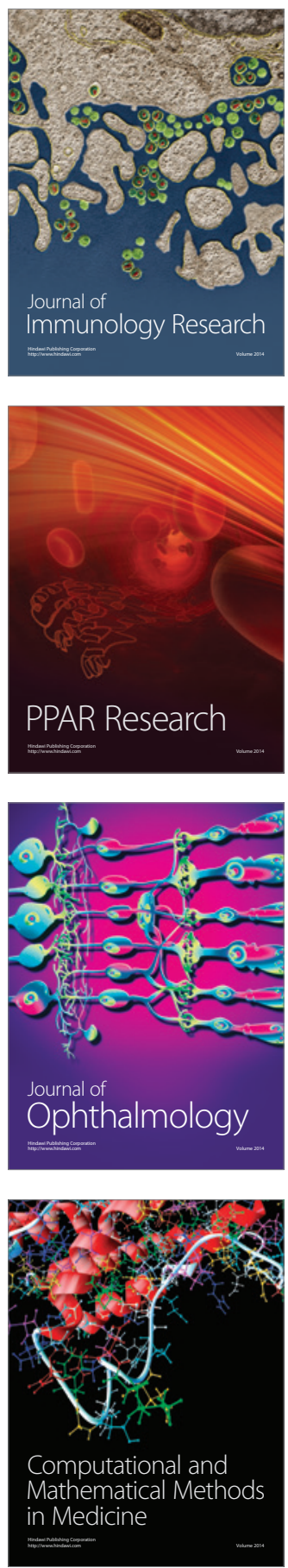

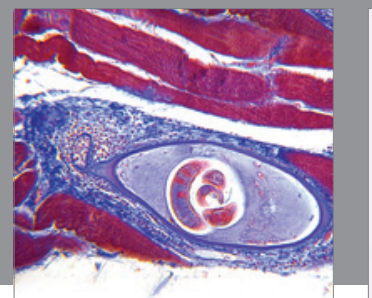

Gastroenterology

Research and Practice
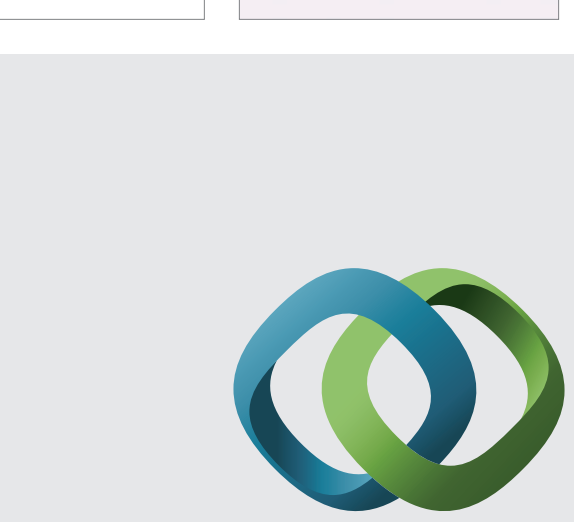

\section{Hindawi}

Submit your manuscripts at

http://www.hindawi.com
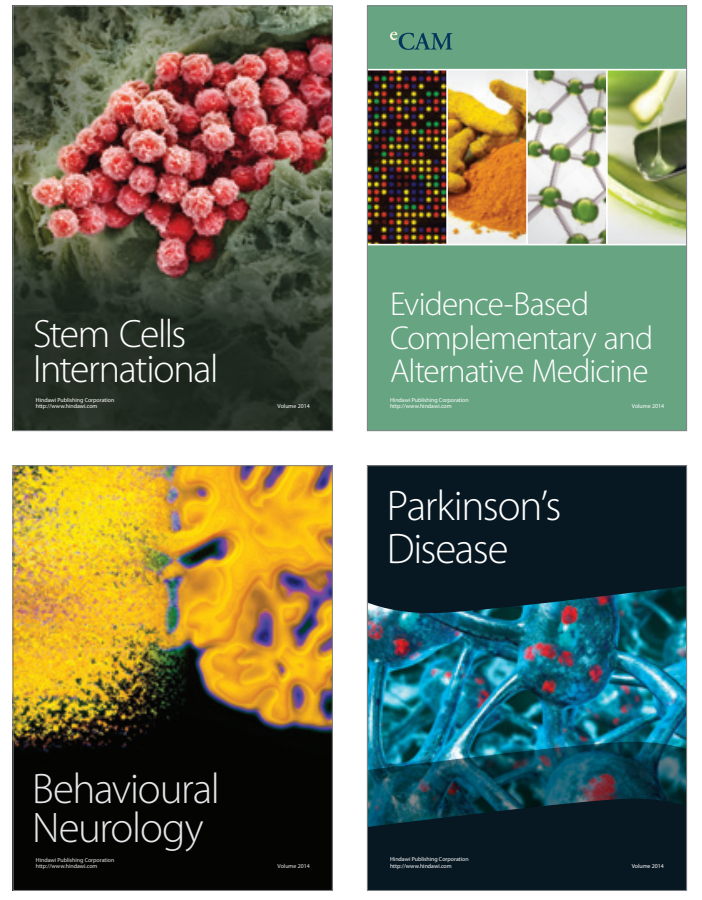
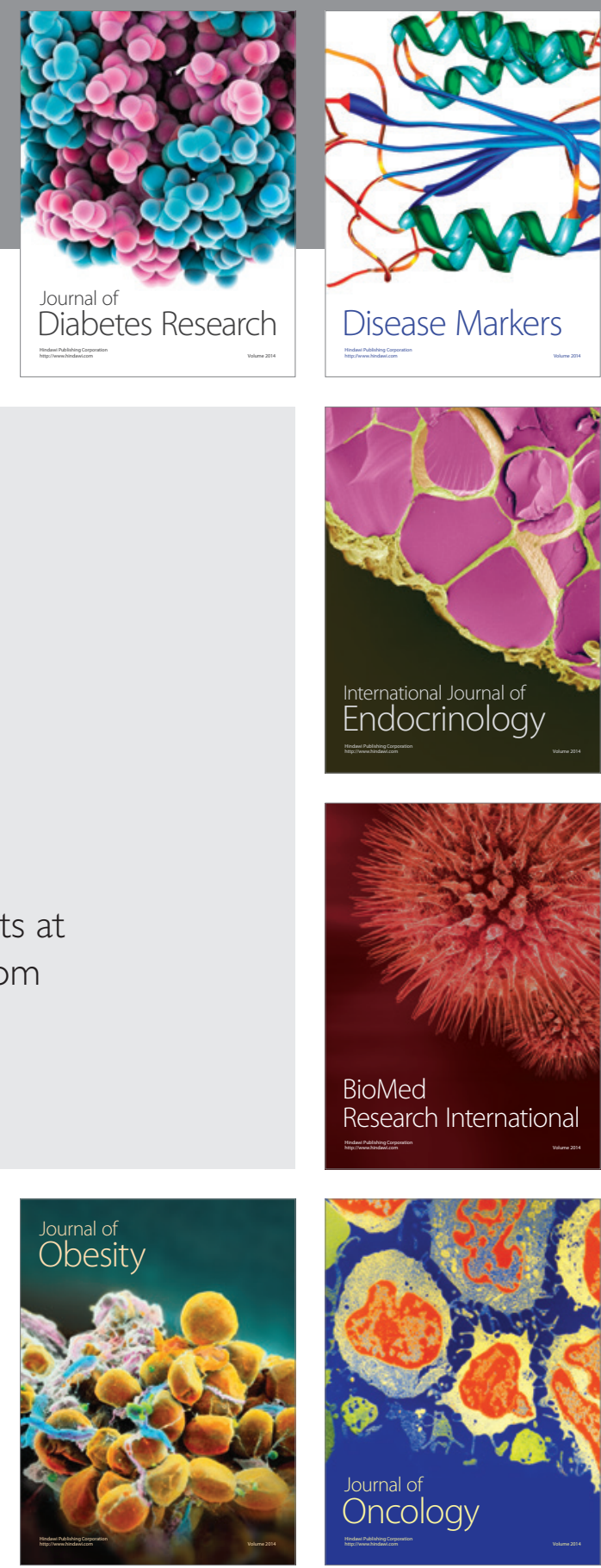

Disease Markers
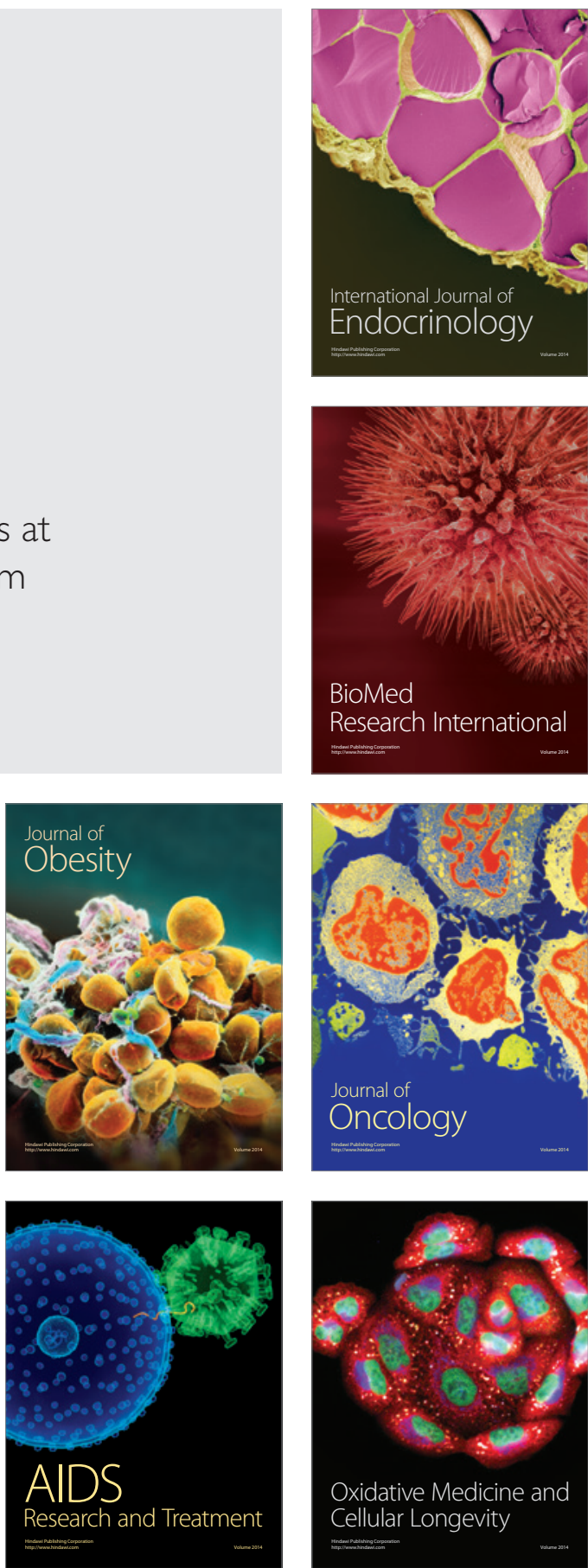